Supplement for

\title{
Global mean surface temperature and climate sensitivity of the EECO, PETM and latest Paleocene
}

Gordon N. Inglis $1,2, \dagger$, Fran Bragg ${ }^{3}$, Natalie Burls ${ }^{4}$, David Evans ${ }^{5}$, Gavin L. Foster 6 , Matt Huber ${ }^{7}$, Daniel J. Lunt ${ }^{3}$, Nicholas Siler ${ }^{8}$, Sebastian Steinig ${ }^{3}$, Richard Wilkinson $^{9}$, Eleni Anagnostou ${ }^{10}$, Margot Cramwinckel ${ }^{11}$, Christopher J. Hollis ${ }^{12}$, Richard D. Pancost ${ }^{1}$ and Jessica E. Tierney ${ }^{13}$

1. Organic Geochemistry Unit, School of Chemistry, School of Earth Science, University of Bristol, UK

2. Cabot Institute for the Environment, University of Bristol, UK

3. School of Geographical Sciences, University of Bristol, UK

4. Department of Atmospheric, Oceanic and Earth Sciences, George Mason University, USA

5. Institute of Geosciences, Goethe University Frankfurt, Frankfurt am Main, Germany

6. School of Ocean and Earth Science, University of Southampton, UK

7. Department of Earth, Atmospheric, and Planetary Sciences, Purdue University, USA

8. College of Earth, Ocean and Atmospheric Sciences, Oregon State University, USA

9. School of Mathematics and Statistics, University of Sheffield, UK

10. GEOMAR Helmholtz Centre for Ocean Research Kiel, Germany

11. Department of Earth Sciences, Utrecht University, Netherlands

12. GNS Science, Lower Hutt, New Zealand

13. Department of Geosciences, The University of Arizona, 1040 E $4^{\text {th }}$ St Tucson AZ USA

${ }^{\dagger}$ present address: School of Ocean and Earth Science, University of Southampton, UK

Corresponding author: Gordon N. Inglis

Email: gordon.inglis@bristol.ac.uk. Telephone: +44 (0)117 9546395 
Figure S1: Predicted surface warming by Gaussian process regression (i.e. $D_{\text {surt }}-3$ ) and associated uncertainties. Anomalies are relative to the present-day zonal mean surface temperature. Circles indicate all available SST and LAT proxy data for the respective time slice that were used to train the model. Circles for locations where multiple proxy reconstructions are available are slightly shifted in latitude for improved visibility
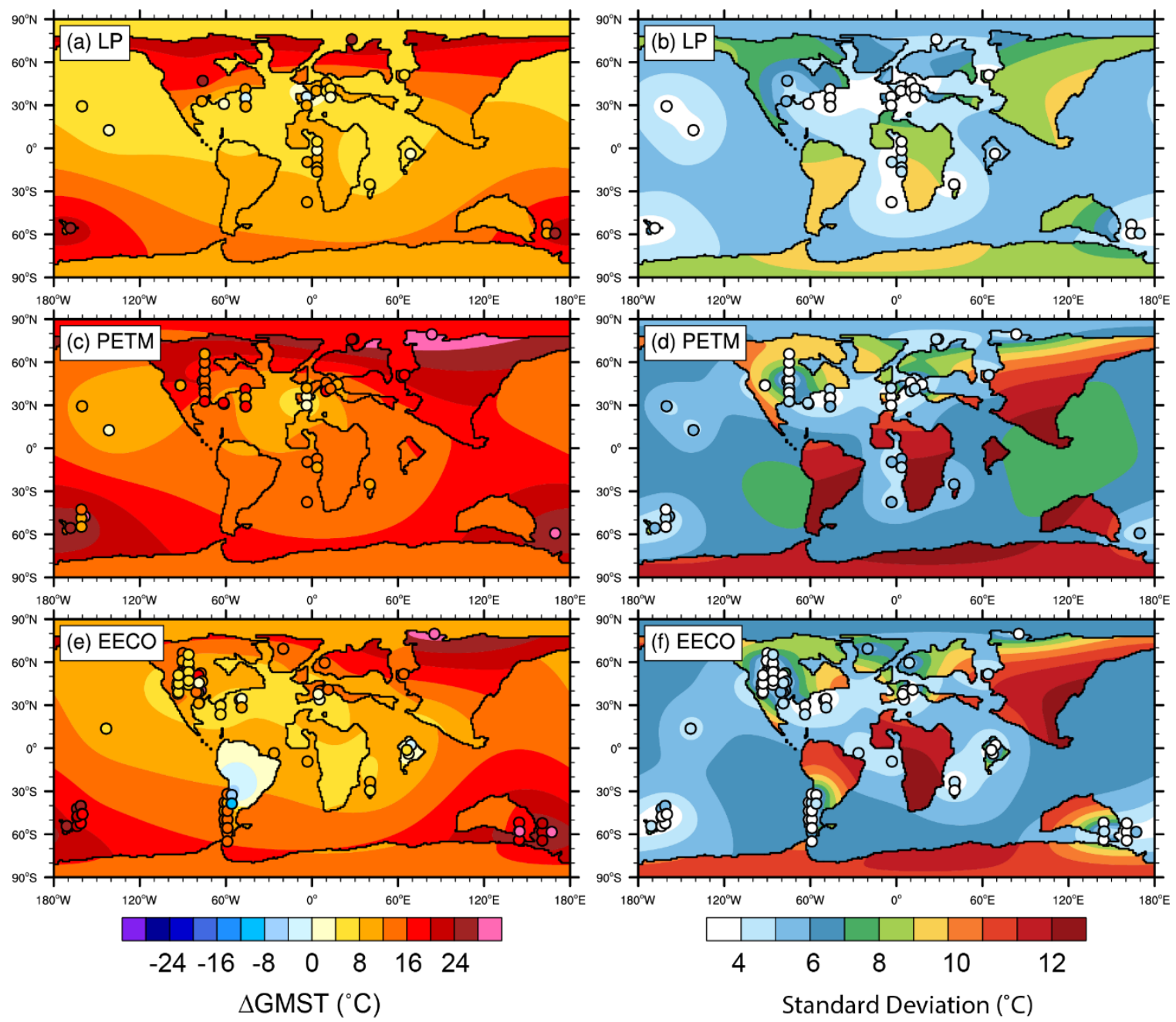
Figure S2: Illustration of $D_{\text {surt }} 4$ comparing zonal-mean near-surface air temperature at sea level, averaged over all months between 1981 and 2000, from ERA5 reanalysis (solid) and Eq. 3 (dashed).

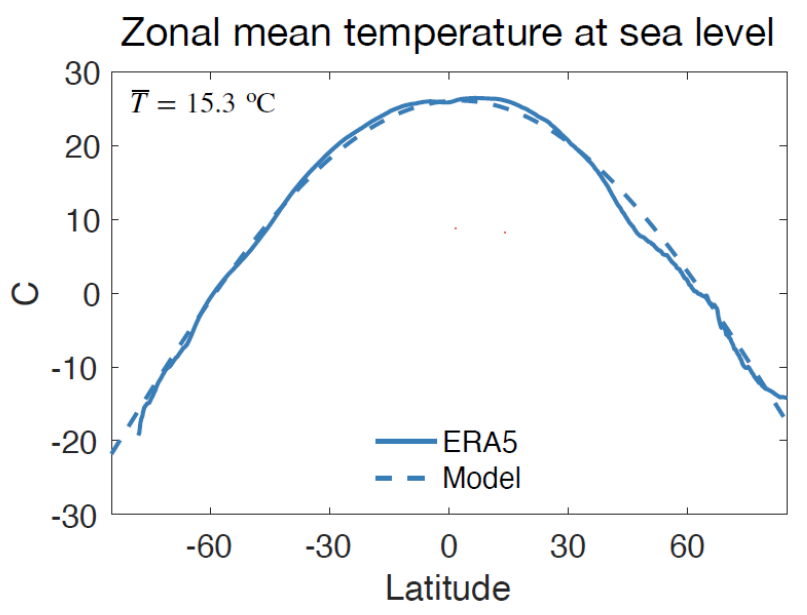


Figure S3: Illustration of $D_{\text {surt }} 4$ illustrating the average (red line) and $90 \%$ confidence interval (red shading) of zonal-mean temperatures computing from proxy LAT (purple) and SST (green) data from the EECO (left), LP (center), and PETM (right).
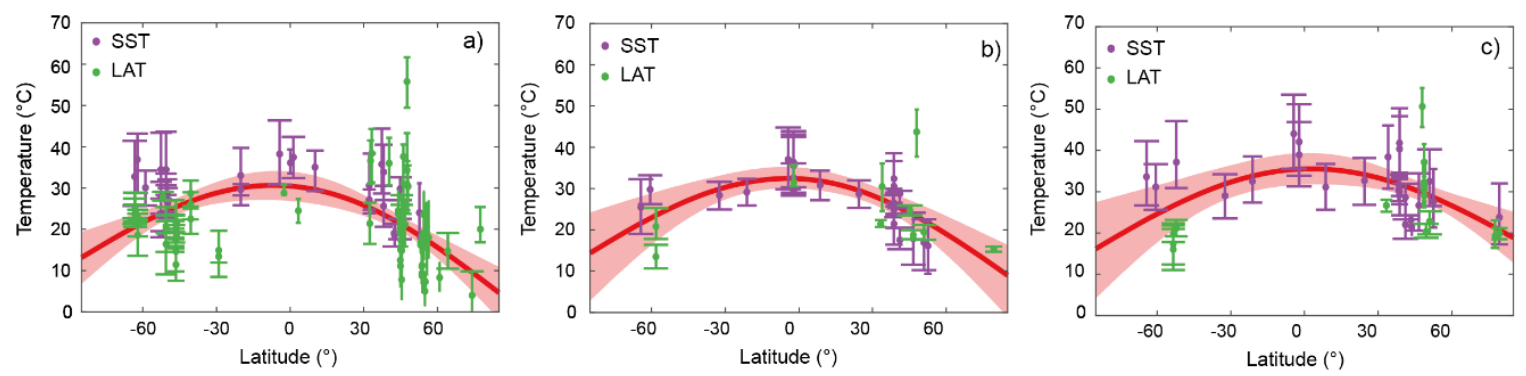